\title{
Pulse-by-Pulse Optical Observation of Growth of Laser-Induced Periodic Surface Structure at Oblique Incidence
}

\author{
Kyosuke Shibata and Shigeki Matsuo* \\ Department of Mechanical Engineering, Shibaura Institute of Technology, Japan \\ *Corresponding author'se-mail: matsuos@shibaura-it.ac.jp
}

\begin{abstract}
An apparatus to record the growing process of laser-induced periodic surface structure (LIPSS) pulse-by-pulse has been constructed. Application of oblique incidence resulted in the formation of LIPSS whose period is longer than the laser wavelength and optical observation of fringes was enabled. Here we show the result of our observations using the apparatus and demonstrate the capability of this method.
\end{abstract}

DOI: $10.2961 /$ jlmn.2020.02.2009

Keywords: laser-induced periodic surface structure, LIPSS, ripple, optical observation, pulse-bypulse, oblique incidence, scratch

\section{Introduction}

Laser induced periodic surface structure (LIPSS, also referred to as ripples) is attracting interest not only because of its capability for surface nanostructuring of solid materials, but also because it is a unique showcase of light-matter interaction in micro/nano space [1], [2]. Formation of LIPSS was firstly reported by Birnbaum in 1965 [3]. The mechanism of the formation of LIPSSs had been understood as the interference between the incident laser beam and the surface scattering wave [4], [5]. In the last two decades, however, LIPSSs whose periods are shorter than those expected in theory have been reported, and the formation mechanism is still under debate.

The mechanism that determines the period of LIPSS is one of the points of interest. There are several articles that investigated the pulse number dependence of the period. Most of them showed that the period decreased with increasing pulse number [6], [7], and a theory why the period decreases has been proposed. However, these researches observed different regions of the samples to evaluate the period with different pulse numbers. In such cases, it is impossible to observe "how" the period was changed.

We think it is important to observe the change in morphology in the growing process of LIPSS (including change in the period) in the same region of the sample to elucidate the formation mechanism of LIPSS. Such observation has been reported by Oboňa et al using laser-scanning microscopy [8]. They analyzed the change in surface morphology up to 10 pulses on a Si substrate precisely and quantitatively. Recently Fuchikami et al. reported that they irradiated with fs laser pulse inside the chamber of a scanning electron microscope and observed the surface of Si substrate up to 2000 pulses in every 33 pulses [9].

Compared to these techniques, simple optical observation is faster and thus easier to acquire more data. Its obvious disadvantage is that spatial resolution of optical observation is not adequate with respect to the period. In the present study, we utilized incident angle dependence of the period. It is generally accepted that oblique incidence will yield both long-period $\left(\Lambda^{+}\right)$and short-period $\left(\Lambda^{-}\right)$LIPSS than the period at normal incidence, i.e.,

$$
\Lambda^{ \pm}=\frac{\lambda}{1 \mp \sin \theta}
$$

or

$$
\Lambda^{ \pm}=\frac{\lambda}{\operatorname{Re}[\eta] \mp \sin \theta}
$$

where $\lambda$ is the laser wavelength, $\operatorname{Re}[\eta]$, is the real part of $\eta$, $\eta=\left[\left(\epsilon_{\text {air }} \epsilon_{\text {metal }}\right) /\left(\epsilon_{\text {air }}+\epsilon_{\text {metal }}\right)\right]^{\frac{1}{2}}$ and $\theta$ is the angle of incidence [10], for p-polarization. Although experimental data does not much Eqs. (1) and (2) at elevated angles [10], the existence of $\Lambda^{+}$is well recognized. Under oblique incidence, the growth of the LIPSS was observed pulse-bypulse using in-situ optical microscopy.

\section{Design and implementation of apparatus}

As described above, oblique incidence was applied for the formation of LIPSS for increasing the period of LIPSS in the present study. While both long-period and short-period LIPSSs were generated, the former occupied a larger area. The surface morphology was in-situ observed pulseby-pulse by optical microscopy from an angle perpendicular to the surface.

\subsection{Optical setup}

The overall system is schematically shown in Fig. 1. The sample substrate was placed on a manual three-dimensional (3D) stage, and its surface was set perpendicular to the optical table (perpendicular to $x$-axis). Laser beam was in $x y$ plane and oblique to the sample surface. The laser used was a microchip Nd:YAG laser (PNP-M08010, Teem Photonics) which emits pulses with wavelength of $1.064 \mu \mathrm{m}$ and pulse duration of $0.5 \mathrm{~ns}$.

The surface of the sample was observed using a system that includes an objective lens (OL), a tube lens (TL), and a camera. The OL used was a long-working-distance OL (LDJ50, Shibuya Optical) which has a magnification of 50x, numerical aperture (NA) of 0.55 , working distance of 
$8.2 \mathrm{~mm}$, and infinity-corrected optics. Because the laser was irradiated from outside of the OL, the minimum value of incident angle $\theta_{\min }$ is restricted by the NA of the OL. The condition $\theta_{\min }>\left(\sin ^{-1} N A\right)+\alpha$ must be satisfied, where $\alpha$ is a positive value, roughly $\alpha \sim 15^{\circ}$, depending on the mechanical design of the OL. The incident angle of $60^{\circ}$, applied in the present study, meets this condition. The TL used was an achromatic lens with a focal length of $200 \mathrm{~mm}$ (AC254-200-A, Thorlabs); the focal length was chosen in accordance with the optical design of the OL. For illumination, an epi-illumination tool (YS-93002-CII, Yashima Optical) was placed between the OL and TL to lead the light of halogen lamp to the sample. At the back focal plane of the OL-TL system, the camera (WRAYCAM-NF300, Raymer) was placed. The overall observation system was mounted on a manual 3D stage. To achieve a fine adjustment of focus, a stage with differential micrometer was used along the optical axis ( $x$ direction in Fig. 1).

\subsection{Timing control of image recording}

The images on the laser-irradiated surface need to be recorded synchronizing with the laser pulse emission. For this, both laser pulse emission and camera recording were triggered by the signal from a microcomputer (Arduino compatible). The details are described in the appendix. Before laser irradiation and after irradiation of each pulse, the morphology of the sample was recorded, and the image was transferred to and saved on a personal computer (PC). The repetition rate of this irradiation-image recording cycle was 0.5 $\mathrm{Hz}$ in the present study.

Post image processing (e.g. trimming, adding scale bar, making video) was carried out using a macro of ImageJ to avoid human error (e.g. trimming different regions).

\section{Experimental results and discussion}

In the present study, the sample was home-polished austenitic stainless steel substrate $(18 \% \mathrm{Cr}-8 \% \mathrm{Ni}$, SUS304 in Japanese Industrial Standards). The polarization was p-polarization, and the angle of incidence was $60^{\circ}$. This incident angle was chosen because the period was maximal at this angle in our prior experiment.

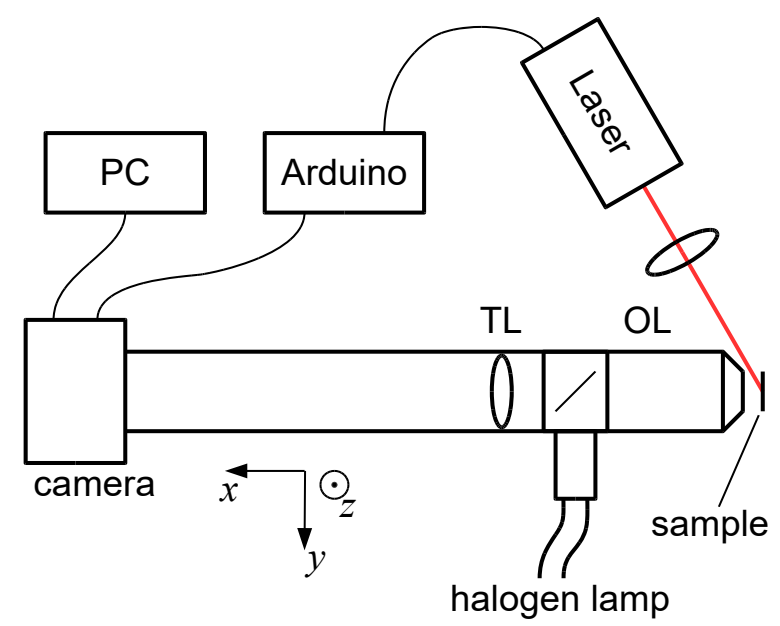

Fig. 1 Scheme of the constructed apparatus. PC: Personal Computer, TL: Tube Lens, OL: Objective Lens.

\subsection{Change in the morphology in the early stage}

Because this method records change in the morphology for all pulses, the change in the early stage, where the most drastic change is expected, can be analyzed. Figure 2 shows the images after irradiation with first three pulses and the image before irradiation.

Scratches are seen in the image before irradiation. The contrast of the scratch marked $\mathrm{P}$, which is in the central region of laser-affected zone, increased after the first laser pulse. This is probably related to the increase in local electric field around a scratch [11]. With subsequent laser pulses, the scratch became less conspicuous. It is probable that surface melting occurred.

The contrast of the scratches marked $\mathrm{Q}$ and $\mathrm{R}$, which are in the peripheral region, also increased after the first pulse.
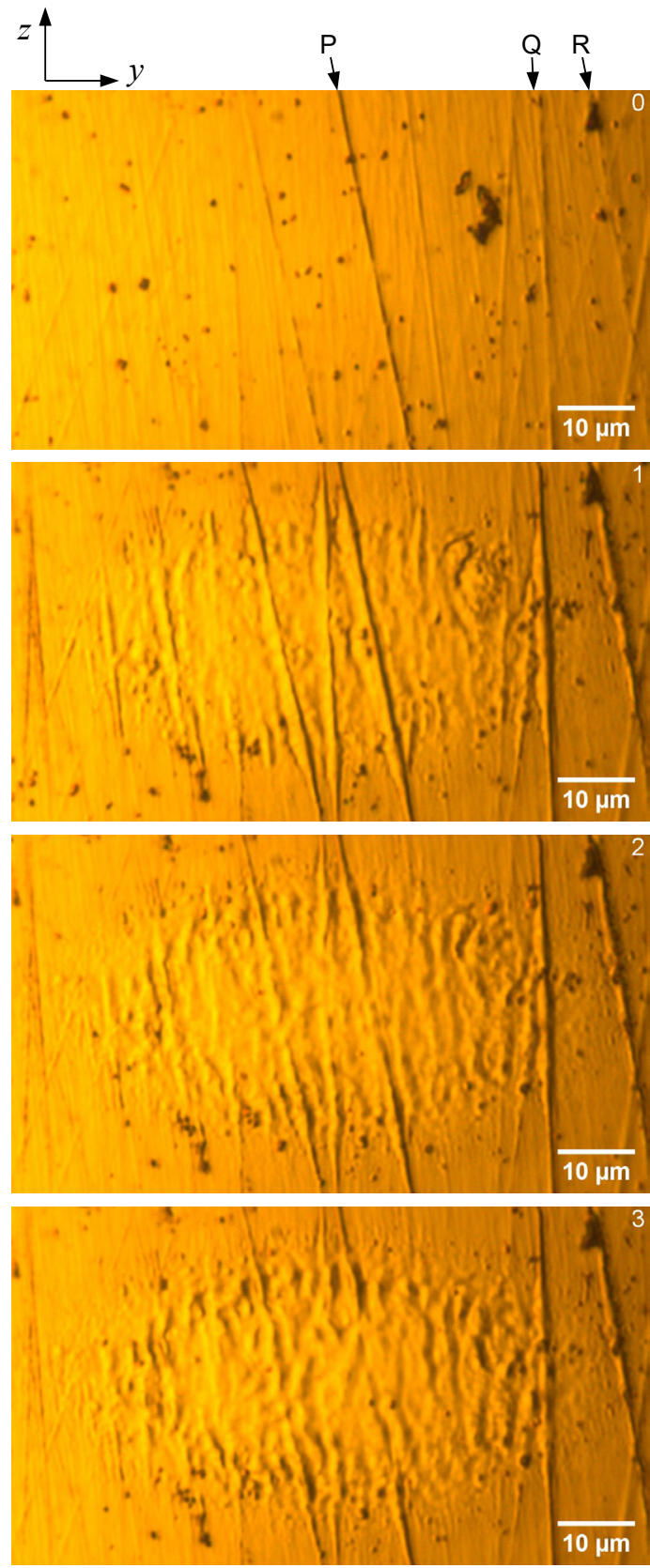

Fig. 2 Change of the image in first three pulses. The number in the upper right corner represents the number of pulses irradiated. 
The increase of contrast was more remarkable than that of P. The left side of the scratches looked brighter than before, while the scratches themselves appeared to be darker. The contrast did not decrease up to three pulses shown here, but they gradually became less conspicuous as in the case of $\mathrm{P}$ with the expansion of laser-affected zone.

Although the direction of the fringes is not so clear, but they generally point to the vertical direction. This is consistent with the general tendency of p-polarized irradiation $[10]$.

\subsection{Change in the size of laser-affected zone}

Generally, the size of the laser-affected zone gradually increases with increasing pulse number. With the present method, the size can be investigated in the same zone. The measured change in size (horizontal width in Fig. 2) was plotted in Fig. 3. Actually, there is a difficulty in judging where the edge of laser-affected zone is, thus the width has systematic error about $\pm 5 \mu \mathrm{m}$, but the random error is much less. In the present case, the size increased up to $\sim 50$ pulses and then saturated, as seen in Fig. 3.

\subsection{Local movement of the fringes}

Even after the size of the laser-affected region reached saturation, the morphology in the region was still changing. Interestingly, the change occurred locally, that is, in some regions the morphology was unchanged, but in some regions the morphology still changed. In Fig. 4, overall image after 143 pulses and magnified images after 143-151 pulses are shown. In the peripherals of each magnified image, the morphology is not changing. However, the morphology in the central region is changing pulse by pulse. The fringes seem to move to the left ( $-y$ direction). Such a local movement has never been observed. We expect this phenomenon to be related to oblique incidence, however, further investigation is required to confirm this finding.

\subsection{Discussion}

In the present study, simple optical microscopy was used for observing the surface morphology. Of course, this method has a disadvantage in resolution against scanning electron microscopy (SEM), atomic force microscopy (AFM), and confocal laser-scanning microscopy (CLSM). The advantage of this method over the others is the speed.

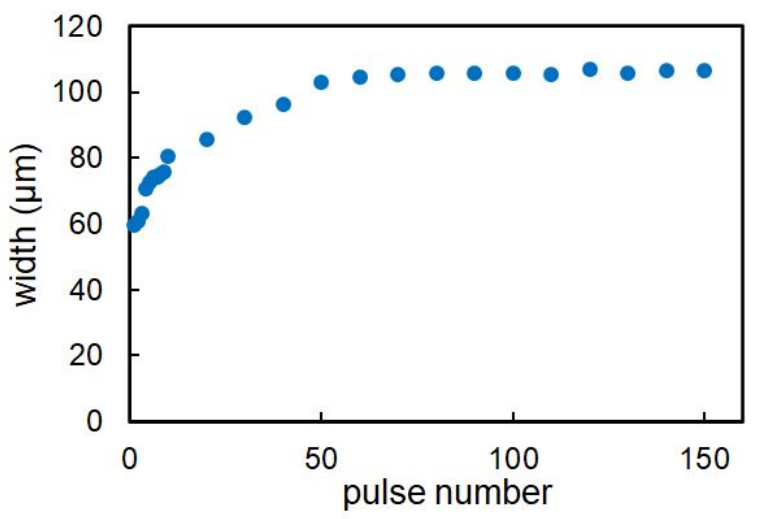

Fig. 3 Change in the lateral size of the laser-affected zone as a function of pulse number.
Although the repetition rate was $0.5 \mathrm{~Hz}$ in the present study, with a faster image-recording system, it is possible to achieve much higher repetition rates, for example, $100 \mathrm{~Hz}$. One more advantage of the present method is there is no displacement of the observing region. This is advantageous in investigating the movement of local patterns. In addition, because there is no restriction on the material of the sample, the present method may be applicable to other materials, e.g., transparent dielectrics.

There is still room of improvements in the current apparatus. One of them is the illumination. The change in contrast observed in Fig. 2 is most probably related to the illumination. By choosing appropriate illumination, and also by acquiring images with different illumination methods, we will be able to get richer information about the surface nanostructures. In addition, implementation of interferometric measurement will allow for quantitative height measurement as well as AFM and CLSM measurements. Speeding up and automating image analysis is also important for extracting information from a large number of images.

\section{Summary and outlook}

We constructed an apparatus to record the change in surface morphology pulse-by-pulse in oblique incidence setup. This was applied to observe the formation process of LIPSS on an austenitic stainless steel substrate, and confirmed to work correctly. Unexpected local movement of the fringes
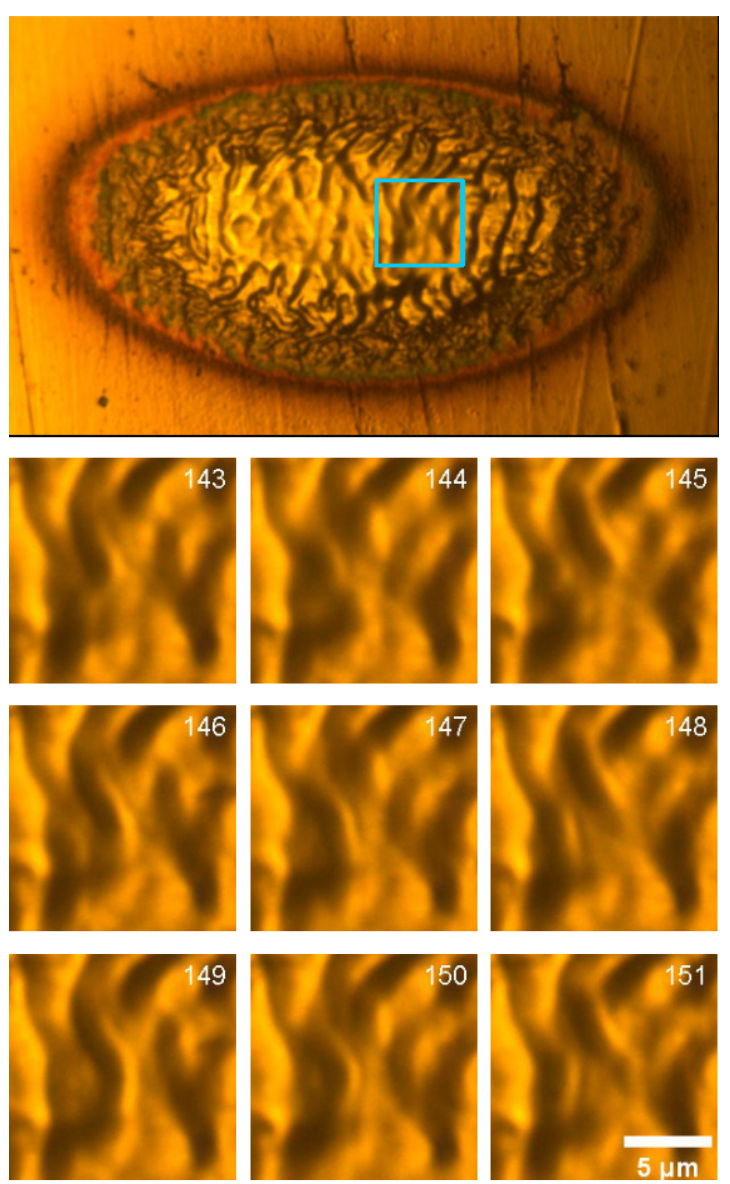

Fig. 4 Overall image at 143-pulse, and local images at $143-151$ pulses. The rectangle in the overall image indicates the position of the local images. 
were observed. This apparatus will be useful for investigating the formation mechanism of LIPSS.

\section{Appendix: Detail of timing control}

The timing chart is shown in Fig. 5. The camera can be triggered by electrical continuity between specific terminals. For this, a relay was used, and a $+5 \mathrm{~V}$ signal was sent to the relay. The laser can be operated directly with a TTL (Transistor-Transistor Logic) signal. For the pulse emission of laser, a $+5 \mathrm{~V}$ signal was sent to the laser from the microcomputer. The times " $400 \mathrm{~ms}$ " and " $2000 \mathrm{~ms}$ " in Fig. 5 are about twice of the minimum times required (the minimum times were determined empirically) for reliable operation. Only the start and end of this process are manually controlled.

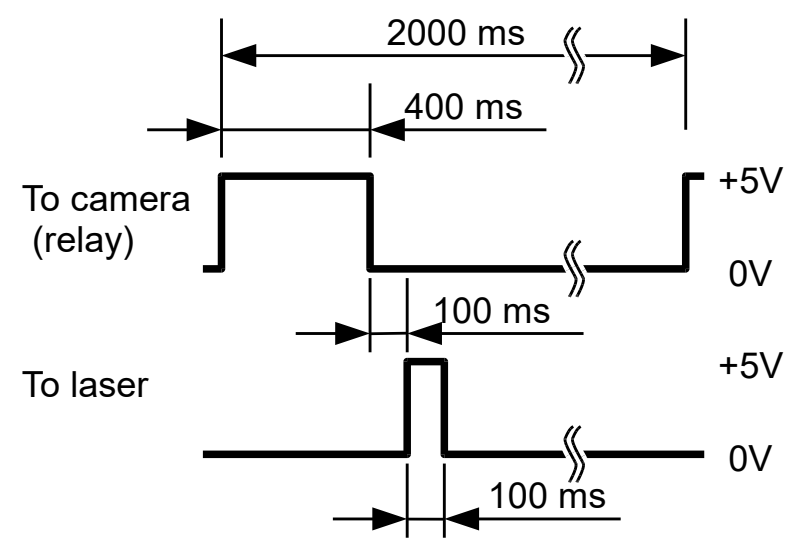

Fig. 5 Timing chart for automatic pulse-by-pulse image recording. This cycle was repeated with a period of $2000 \mathrm{~ms}$.

\section{References}

[1] J. Bonse, S. Hohm, S. V. Kirner, A. Rosenfeld, and J. Kruger: IEEE J. Sel. Top. Quantum Electron., 23, (2017), 9000615.

[2] R. Buividas, M. Mikutis, and S. Juodkazis: Prog. Quantum Electron., 38, (2014), 119.

[3] M. Birnbaum: J. Appl. Phys., 36, (1965), 3688.

[4] J. E. Sipe, J. F. Young, J. S. Preston, and H. M. van Driel: Phys. Rev. B, 27, (1983), 1141.

[5] J. F. Young, J. S. Preston, H. M. van Driel, and J. E. Sipe: Phys. Rev. B, 27, (1983), 1155.

[6] M. Huang, F. Zhao, Y. Cheng, N. Xu, and Z. Xu: ACS Nano, 3, (2009), 4062.

[7] J. Bonse and J. Krüger: J. Appl. Phys., 108, (2010), 034903.

[8] J. V. Oboňa, J. Z. P. Skolski, G. R. B. E. Römer, and a. J. H. in t Veld: Opt. Express, 22, (2014), 9254.

[9] Y. Fuchikami et al.: Extended abstracts of the 80th The Japan Society of Applied Physics Autumn Meeting, (2019), 18p-N304-8. (in Japanese)

[10] Y. Fuentes-Edfuf, J. A. Sánchez-Gil, C. Florian, V. Giannini, J. Solis, and J. Siegel: ACS Omega, 4, (2019), 6939.

[11] P. Temple and M. Soileau: IEEE J. Quantum Electron., 17, (1981), 2067.

(Received: July 17, 2020, Accepted: September 3, 2020) 